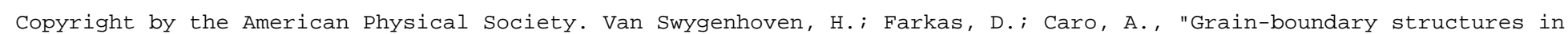
polycrystalline metals at the nanoscale," Phys. Rev. B 62, 831 DoI: http://dx.doi.org/10.1103/PhysRevB.62.831

PHYSICAL REVIEW B

VOLUME 62, NUMBER 2

1 JULY 2000-II

\title{
Grain-boundary structures in polycrystalline metals at the nanoscale
}

\author{
H. Van Swygenhoven \\ Paul Scherrer Institut, CH-5232 Villigen PSI, Switzerland
}

D. Farkas

Department of Materials Science and Engineering, Virginia Polytechnic Institute and State University, Blacksburg, Virginia 24061-0237

A. Caro

Centro Atómico Bariloche, 8400 Bariloche, Argentina

(Received 10 November 1999; revised manuscript received 20 March 2000)

\begin{abstract}
We present a detailed analysis of grain-boundary structures in computer-generated $\mathrm{Cu}$ and $\mathrm{Ni}$ threedimensional nanocrystalline samples. The study includes both totally random and textured microstructures with grain sizes in the range of 5-12 nm. A detailed direct visualization technique is used at the atomic scale for studying the grain-boundary structural features. The study focuses on determining the presence of regions in the boundary exhibiting order and structural units normally expected for high-angle boundaries. For low-angle boundaries we investigate the presence of dislocation networks accommodating the misfit between the grains. A significant degree of crystalline order is found for all the boundaries studied. The highest degree of structural order was identified for boundaries with misfits within about $10^{\circ}$ deviation from the perfect twin. These grain boundaries contain a repeated building structure consisting of structural units typical of a $\Sigma=3$ symmetrical tilt twin boundary and highly disordered steps between those structural units. For all other types of misfit, we also observe some degree of structural coherence, and misfit accommodation occurs in a regular pattern. The cases studied include grain boundaries with a high-index common axis and show structural coherency that is independent of the grain size. Similar results are obtained for textured samples containing only low-angle grain boundaries, where regular dislocation arrays that are typical of larger grain materials are observed. These results provide evidence against the view of grain boundaries in nanocrystals as highly disordered, amorphous, or liquidlike interfaces. The results suggest that the grain-boundary structure in nanocrystalline materials is actually similar to that found in larger grain polycrystals.
\end{abstract}

\section{INTRODUCTION}

The reduction of the grain size down to the nm regime has opened new and fascinating avenues for research in several aspects of materials science. At the lower end of the grain size range obtainable nowadays, about half of the atoms belong to, or are affected by, the presence of interfaces. Grain boundaries are believed to play a predominant role in the plastic deformation of such materials, although the details of how precisely deformation occurs are still uncertain.

The microstructure and properties of the nanocrystalline materials are strongly dependent on the fabrication procedure (for example, pulsed electrodeposition, severe plastic deformation, ball-milling, or inert gas condensation). ${ }^{1-5}$ Some of the fabrication methods have a tendency to produce samples with a significant degree of crystallographic texture, whereas for other procedures (usually those where the precursor is a nanopowder) texture is not observed.

If the grains in a nanocrystalline sample are randomly oriented, low-angle boundaries are expected to be rare and it is therefore reasonable to assume that most boundaries are large-angle boundaries. In coarse grains and for some large angles, coincidence site lattice (CSL) boundaries develop, ${ }^{6}$ which can be viewed as particularly low-energy arrangements of grain-boundary dislocations. ${ }^{7}$ For this kind of boundaries, a detailed description is more difficult. The quantitative dislocation model of crystal boundaries cannot be applied for misorientation angles exceeding about $15^{\circ}$ because the dislocation spacing becomes small enough for the cores to overlap. In that case, the energy of the boundary may approach a constant (relatively high) value independent of the angle, with cusps in energy corresponding to some special coincident site lattice boundaries. It is still under debate what the atomic arrangement in general boundaries is. Standard textbooks suggest that one possibility is that there is an alternation of coherent regions and noncoherent regions, whose shape and size would depend on the misorientation. ${ }^{8}$ The structure of boundaries with misorientations that are in the vicinity of a certain coincident site lattice boundary can exhibit regions in their structure that are similar to that of a coincidence site boundary and other regions that can be more disordered. The actual position of the atoms in these latter regions might be even random or liquid like. Another possibility is that the misfit region between neighboring crystals resembles that of adjacent regions of short-range order in liquids, and the boundary can be viewed as a liquid monolayer boundary. The excess energy per atom involved in such a high-angle boundary is then approximately equal to the latent heat of fusion. Research on ideal, planar bicrystal interfaces has been enriched by the contribution of combined computer simulations and careful experiments. $^{9-14}$

Controversial results have been reported for the structure 
of grain boundaries in nanocrystalline materials. ${ }^{15-21}$ Two extreme pictures have been proposed: the earliest, supported by some experiments and computer simulations, ${ }^{22}$ suggests a nonequilibrium, highly disordered "frozen-gaslike" grain-boundary structure, substantially different from structures in coarse-grained polycrystalline materials. More recent experiments suggest that grain boundaries in nanocrystalline materials are not anomalous, but similar to those found in polycrystalline materials. The studies in the literature use different measuring techniques, which are often not directly comparable. Another problem is the different synthesis techniques and different aging and annealing histories used in the various experimental studies. A considerable effort both in refining experimental techniques such as X-ray diffraction and absorption measurements and high-resolution electron microscopy, and in comparing different synthesis techniques is required to clarify this controversy.

For some simple materials for which reliable interatomic potentials exist, molecular dynamics computer simulations provide an atomistic view of the microstructure through the mean-field approximation of atomic interactions. Several "fabrication" procedures for nanocrystalline metals have been explored, which can be characterized by the degree of relaxation that is allowed in the synthesis process. In the case of the sintering of two isolated $\mathrm{Cu}$ nanoparticles, a case of minimum imposed constraints, Zhu and Averback ${ }^{23}$ showed that the large surface-to-volume ratio generates strong enough driving forces to induce rotation, plastic deformation, and densification. As a consequence, a low-angle grain boundary results even for random initial misorientation. Introducing more constraints, simultaneous sintering of several Pt particles at different applied pressures was analyzed by Liu et al. $^{24}$ They report several phenomena such as surface rounding, neck formation, void formation and shrinking, and cluster extrusion. The resulting grain boundaries are very narrow and exhibit only localized disorder. Imposing even more constraints, Phillpot and Wolf ${ }^{25}$ studied the synthesis of a fully dense Lennard-Jones nanocrystalline material grown by crystallization from the melt. The sample was designed to have eight crystalline seeds with particularly chosen orientations such that well-defined high-angle boundaries were formed. They found that these boundaries differ fundamentally from those well known from bicrystal studies. In particular, they observed low-density regions in the grain boundary, absence of long-range periodicity, narrower grainboundary energy distribution, and larger grain-boundary width, with a narrow distribution as compared to bicrystals. Based on these simulations, they suggested a simple structural model for grain boundaries in nanocrystalline materials based on a "cementlike", phase, reminiscent of Rosenhain's amorphous-cement model. ${ }^{26}$

In our previous work ${ }^{27-30}$ we performed detailed simulations of uniaxial tensile deformation in $\mathrm{Cu}$ and $\mathrm{Ni}$ model nanophase samples, with grain sizes of 3.4-12 nm, in a temperature range of $300-500 \mathrm{~K}$ and with uniaxial tensile stress from 0.05 to $1.5 \mathrm{GPa}$. We analyzed the behavior of two classes of boundaries: completely random (mostly high angle) and textured (with misorientations restricted to below $17^{\circ}$, low angle). Intergrain sliding as well as intragrain dislocation activity was observed as a function of grain size and stacking fault energy, with sliding being the only mechanism operating for the smallest grain sizes explored. To interpret these observations at small grain size (sliding regime), we assumed that macroscopic displacement is the result of grains sliding against each other, obeying a general nonlinear viscous behavior with standard thermal- and stress-assisted activation processes. The activation energy and volume indicate that the elementary microplastic event is an atomic jump in a disordered region. The determination of the actual grainboundary structure as a function of grain size is critical to understanding the deformation mechanism in the nanophase samples.

In the present work, we focus on the study of the interfaces responsible for the plastic deformation, aiming at providing a structural characterization of them. We report a detailed analysis of the microstructure of several relaxed, undeformed, nanophase samples, namely, Ni $12 \mathrm{~nm}$, Ni 8 $\mathrm{nm}, \mathrm{Ni} 5.2 \mathrm{~nm}, \mathrm{Cu} 12 \mathrm{~nm}$ random high angle (HA) and $\mathrm{Cu} 8$ $\mathrm{nm}$ textured low angle (LA).

\section{COMPUTATIONAL TECHNIQUES}

We use two different procedures to create the nanocrystalline samples based on unconstrained and constrained stochastic methods. In the first one, the simulation cell volume is filled with nanograins grown from seeds with random location and crystallographic orientation; the space is filled until the grains overlap each other, according to the Voronoi construction. ${ }^{31}$ In the second procedure, the grain misorientations are still chosen at random, but constrained to be less than a given angle $\left(17^{\circ}\right.$ in this case). We refer to these procedures as HA and LA samples, respectively. The Voronoi procedure gives irregular Wigner-Seitz polyhedra, whose faces, the grain boundaries, result randomly oriented as well. The samples are then relaxed for $50-100 \mathrm{p}$ at $300 \mathrm{~K}$ using molecular dynamics, giving a metastable equilibrium state with final density between $96 \%$ and $97 \%$ of the perfect crystal value. Further relaxation at this temperature does not change the overall density and does not induce significant grain growth or rotation. The density is related to the grainboundary type and mean grain size and is quite insensitive to the relaxation procedure. Simulations done under hydrostatic pressure up to $10 \mathrm{GPa}$ and $500 \mathrm{~K}$ followed by relaxation still do not produce an increase in density ${ }^{32}$

In order to study the effect of grain size, the Ni $5.2 \mathrm{~nm}$ and the Ni $12 \mathrm{~nm}$ HA samples were constructed using the same set of random locations and orientations. The same microstructure appears in both samples with boundaries that have exactly the same misorientations and orientation of the grain-boundary planes. These samples differ only in their scale, that is, the number of atoms, and therefore the grain size. This comparison allows us to study the same grain boundary for different grain sizes, isolating the effect of grain size on grain-boundary structure.

As a description of the interatomic interaction, we use the second moment (tight-binding) potentials for $\mathrm{Ni}$ and $\mathrm{Cu}$ (Ref. 33) in the Parrinello-Rahman ${ }^{34}$ approach. In order to assess the dependence of our results on the interatomic potentials used, we have also tested samples relaxed using two other interatomic potentials. The first one is the embedded atom method (EAM) potential for Ni developed by Baskes et $a l .{ }^{35}$ The second one is a more recent interatomic potential 
for $\mathrm{Ni}$ developed on the basis of ab initio calculations and also using the EAM framework. ${ }^{36}$ The latter potentials accurately reproduce many experimental properties, including the stacking fault energy as well as $a b$ initio calculations of structural energies of metastable configurations. This latter feature helps ensure that the potential will perform well in situations far from equilibrium. We choose $\mathrm{Cu}$ and $\mathrm{Ni}$ as model materials because they have very different stacking fault energies and therefore enable us to study the effect of stacking fault energy on the observed grain-boundary structure.

We use periodic boundary conditions in all three directions and sample sizes that are between $10^{5}$ and $10^{6}$ atoms. The molecular dynamics computational procedure used is described in more detail in our previous work. ${ }^{28,30}$

The selection of the boundaries studied was done based on the overall microstructure and included the boundaries that seemed the most ordered as well as those that seemed the most disordered. Since it was not possible to analyze all the boundaries present, we started with a two-dimensional visualization of cross sections of the sample every $0.5 \mathrm{~nm}$ and chose a number of representative boundaries. The selected grain boundaries represent different misorientations between the grains, ranging from $9^{\circ}$ to $75^{\circ}$.

As an analysis tool, we investigate the coordination number and type of each atom in the sample using the topological medium-range-order analysis developed by Honneycutt and Andersen, ${ }^{37}$ which is based in the classification of atom pairs. This technique is based on determining the configuration of the common neighbors of a selected atom pair and associating each possible configuration with a four-digit number. The first digit indicates whether the atoms in the selected pair are nearest neighbors (1) or not (2). The second digit is the number of the common nearest neighbors of the pair, while the third digit counts the number of bonds among the common nearest neighbors Finally, the last digit tells us the number of bonds in the longest continuous path that goes through the common neighbors. The above procedure provides a distinction between fcc and hcp structures, even taking into account only nearest-neighbor pairs. An arbitrary atom in a perfect fcc structure forms only 1421-type pairs with its 12 nearest neighbors, while in the case of the hcp lattice one would find six 1421 and six 1422 pairs. Using this analysis, we define four color-coded categories of atoms: gray symbols represent atoms with local fcc order, red symbols represent atoms with local hcp order, green symbols represent atoms with other 12-coordinated combinations, and finally blue ones refer to non-12-coordinated atoms. This tool proved to be very important in the visualization of the grain-boundary structures and is helpful in identifying regions typical of a twin boundary as well as regions including a stacking fault that may result from dislocation dissociation into Shockley partials.

In our technique, we compare the structural properties of the same grain boundary in a 5.2-nm sample and in the $12-\mathrm{nm}$ sample, to investigate the influence of the grain size. In the following section, we first show examples of grain boundaries in the $\mathrm{Ni}$ and $\mathrm{Cu} \mathrm{HA}$ samples, and then we focus on the $\mathrm{Cu} 8$-nm LA sample.

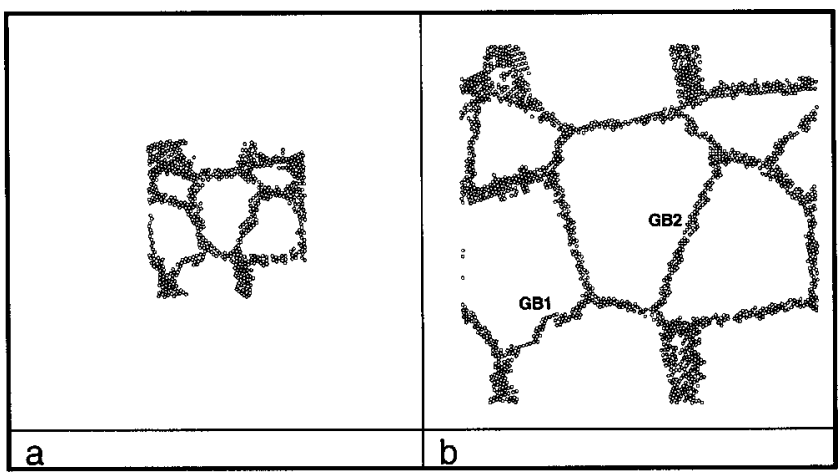

FIG. 1. Cross sections of Ni 5.2-nm HA sample (a) and Ni 12-nm HA sample (b). Only atoms with non-fcc crystalline order are represented. The samples have similar microstructure, but different average grain sizes.

\section{RESULTS}

\section{A. High-angle $\mathrm{Ni}$ and $\mathrm{Cu}$ samples}

Figure 1(a) shows a cross section of the Ni 5.2-nm HA sample. Only atoms with non-fcc crystalline order are represented. The corresponding slice in the larger grain sample Ni 12-nm HA is given in Fig. 1(b). Both samples have the same number of grains with exactly the same orientation for each grain. These cross sections show that the samples have similar microstructure, but different average grain sizes. The larger grain sample has a microstructure that is a scaled-up version of the microstructure of smaller grain sample. Therefore, all the grain boundaries in the small grain sample have a corresponding boundary in the larger grain sample with the same set of misorientation parameters and equivalent orientation of the grain-boundary plane. The only difference between these samples is the grain size. In Fig. 1(b) we have indicated two of the grain boundaries analyzed, denoting them by GB1 and GB2. For all of them, we prepared smaller sections of the full samples to be able to visualize the structure in detail at an atomic level. Similarly oriented sections are cut in Ni 12-nm HA and Ni 5.2-nm HA samples for comparison.

The color in subsequent figures is used to distinguish the four types of atoms defined after the Honneycutt-Andersen analysis: gray atoms are fcc atoms, red atoms are hcp coordinated, green atoms are other 12-coordinated atoms, and blue atoms represent coordination other than 12 . We note that in the fcc structure, the presence of two consecutive (111) planes with hcp-coordinated atoms indicates a stacking fault. Similarly, the presence of a single (111) plane of hcp atoms corresponds to a twin. Therefore these two types of defects can be easily identified in our technique.

Figure 2 shows the grain boundary in Ni 12-nm HA, identified as GB1 in Fig. 1. Figure 2(a) is a view perpendicular to the grain-boundary plane, and Fig. 2(b) is a view of the boundary plane itself. The grain boundary appears as a sequence of structural building blocks, each one formed by a portion of a (111) twin boundary plane (red atoms) and a step between them, which is a disordered region (blue/green atoms). The presence of these disordered regions is related to the misorientation itself and, as we show below, is independent of grain size. This twin-and-step block is repeated several times, forming a stair with the twins in parallel, but not 


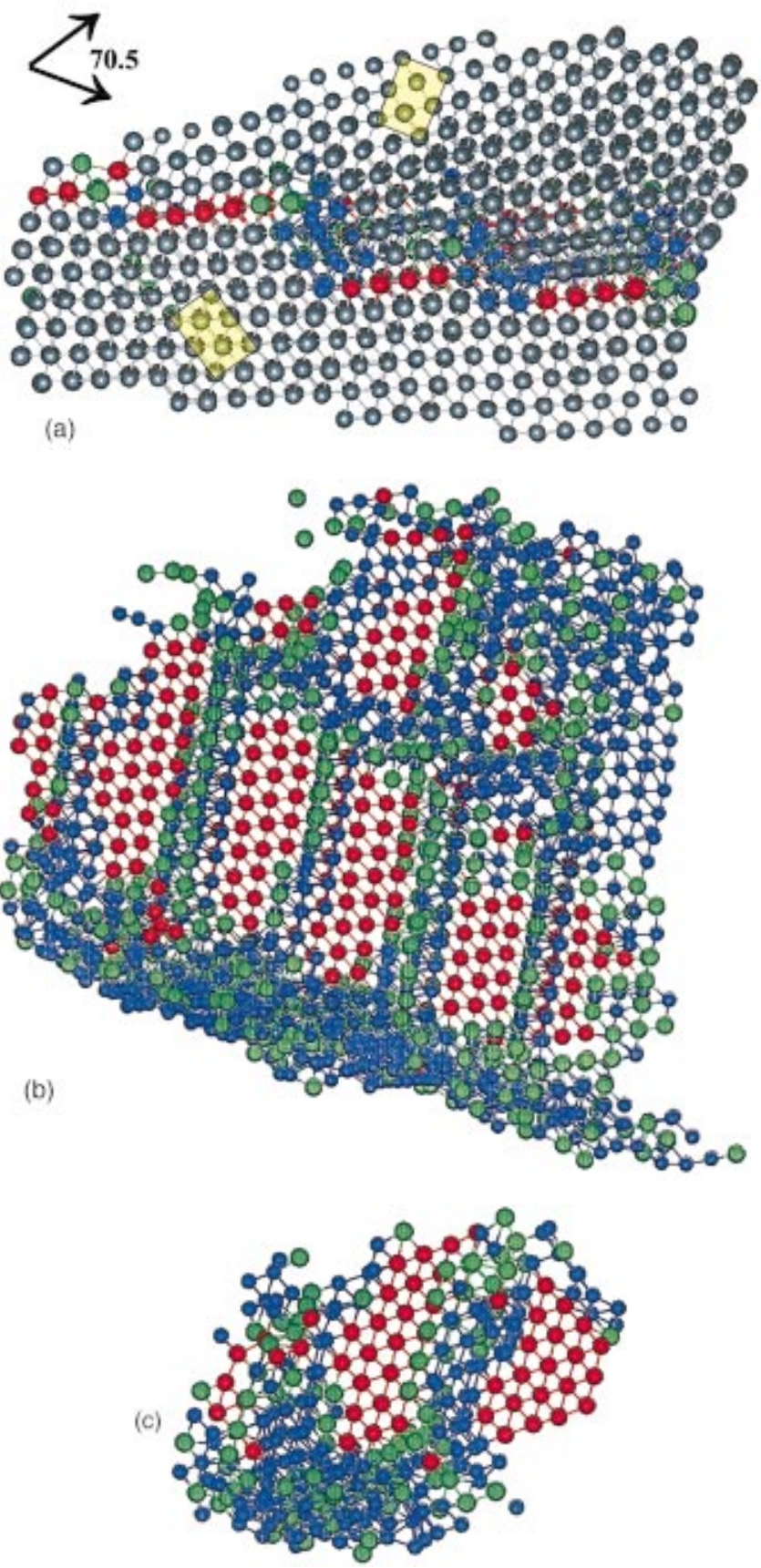

FIG. 2. (Color) The grain boundary in the Ni 12-nm HA sample, identified as GB1 in Fig. 1. (a) View perpendicular to the grainboundary plane, along a common axis, which is close to a $\langle 110\rangle$ direction. (b) View of the grain-boundary plane. (c) The corresponding boundary in the Ni 5.2-nm HA sample. Gray symbols represent atoms with local fcc order, red symbols represent atoms with local hcp order, green symbols represent atoms with other 12-coordinated combinations, and blue ones refer to non-12coordinated atoms.

consecutive (111) planes. The misorientation between grains in this boundary is $75^{\circ}$, which is close to a $\Sigma=3$ coincidence lattice, $70.5^{\circ}$. The view in Fig. 2(a) is along a common axis which is close to a $\langle 110\rangle$ direction. The unit cells are highlighted in both grains. The size of the complete structural block (twin region plus step) shown in plane view in Fig. 2 (b) is about 5.5 times the distance between $\langle 110\rangle$ planes, or approximately $1.4 \mathrm{~nm}$. The block is repeated 5 times along a
$\{111\}$

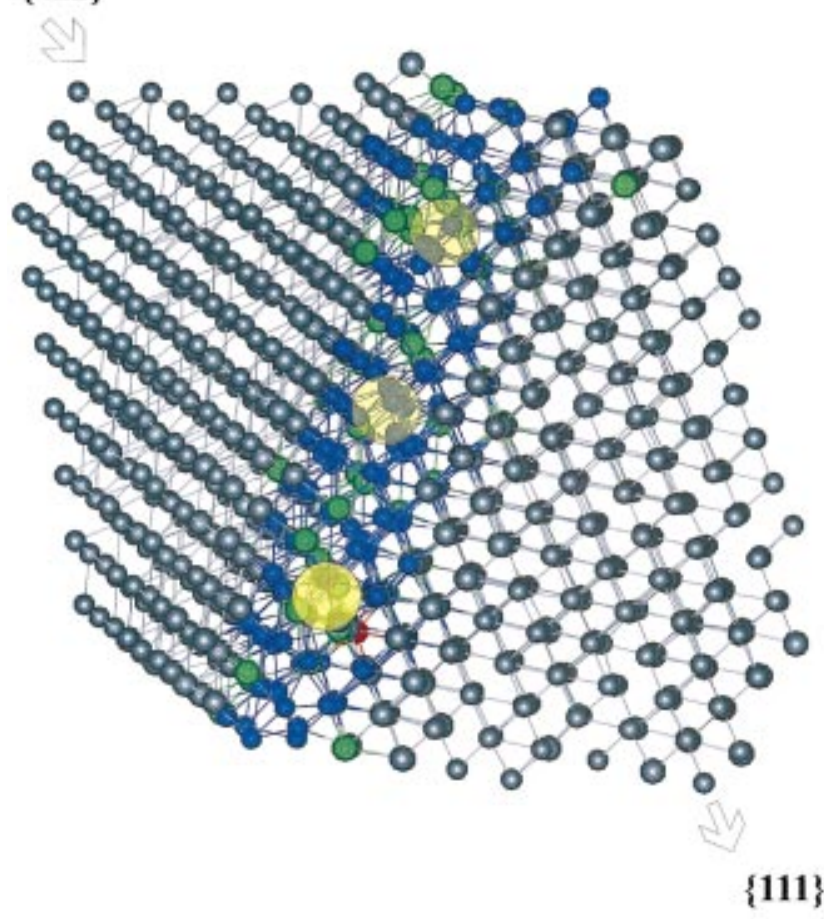

FIG. 3. (Color) The grain boundary in the Ni 12-nm HA sample, identified as GB2 in Fig. 1. The misorientation between grains in this boundary is $75^{\circ}$. View along a noncommon $\langle 110\rangle$ direction for the grain on the right side of the figure. The $\{111\}$ planes of the grain on the left grain continue onto $\{311\}$ planes of the grain at right. The color code is the same as in Fig. 2.

direction close to the $\langle 112\rangle$ direction, making a linear dimension of $\sim 6.8 \mathrm{~nm}$. The size of the entire grain boundary between these particular two grains has maximum dimensions of $7.0 \mathrm{~nm}$. Therefore, our observations suggest that the influence of the triple junction on the grain-boundary structure is short ranged, extending only a few angstroms from its center. We note that the stair structure is a way to accommodate the approximately $12^{\circ}$ difference between the common (111) plane and the actual boundary plane, as well as the $4.5^{\circ}$

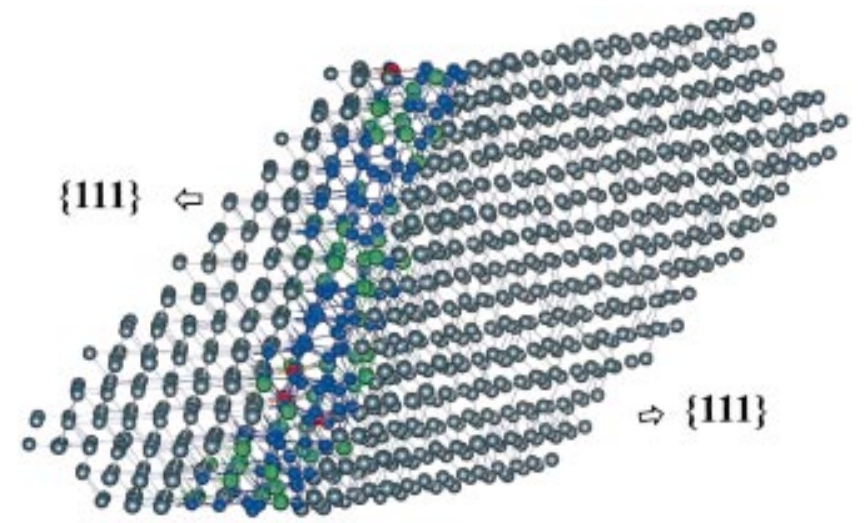

FIG. 4. (Color) The grain boundary labeled GB4 in Ni 12-nm HA, with a high-index common crystallographic axis. The left grain is viewed along a $\langle 110\rangle$ axis. Significant structural coherency across the boundary can also be observed in this case. The color code is the same as in Fig. 2. 
difference between the perfect twin misorientation and the actual misorientation for this boundary.

The corresponding section in the Ni 5.2-nm HA sample, Fig. 2(c), shows a similar structural block as found in the $12-n m$ sample. In this case, however, there is no clear repetition of this unit due to the restriction imposed by the smaller grain size. Even though the tendency to form a portion of a perfect twin is evident, the maximum size of this particular grain boundary is approximately $2 \times 3 \mathrm{~nm}^{2}$. It is clear that in this small grain-boundary plane further repetition of the structural block is not possible. Taking into consideration the size of the structural block, the range of influence of the triple junction for this particular grain boundary is again very short. We see then that the structural features of the boundary are basically independent of grain size in this range.

We also studied a second boundary [GB3, not indicated in Fig. 1(a)] that is similar to the one described above. It has a misorientation of $65^{\circ}$ about an axis close to $\langle 110\rangle$. This misorientation is about $5^{\circ}$ smaller than that of a perfect twin. The structure also contained structural blocks corresponding to a perfect $\Sigma=3$ twin misorientation, very similar to those observed in Figs. 2(a)-2(d). The block was also observed in the 5.2-nm sample. Both of these grain boundaries GB1 and GB3 can be considered as special $\Sigma=3$ boundaries, since the deviation from the perfect $\Sigma=3\left(70.5^{\circ}\right.$ misorientation $)$ is within $15 /(3)^{1 / 2}$, as per the Brandon criterion. ${ }^{38}$ In agreement with this criterion, our study confirms that these boundaries present a significant fraction constituted by structural units typical of the perfect $\Sigma=3$ boundary. The critical observation of the present work is that this expected structure actually holds for the very small grain sizes tested here.

We studied the differences in the grain-boundary structure of this particular boundary in $\mathrm{Cu} 12 \mathrm{~nm}$ as opposed to $\mathrm{Ni} 12$ $\mathrm{nm}$. The $\mathrm{Cu}$ sample for this study has the same set of random crystalline rotations and locations, differing from the $\mathrm{Ni}$ 12-nm HA sample only in the lattice parameter and the interatomic potential. The purpose of this comparison was to assess the influence of the stacking fault energy on the grainboundary structure. We found that the basic structure of the boundaries is the same for both model materials and is then mainly determined by the relative crystallographic orientation of the grains. The figures are omitted for brevity.

In Fig. 3 we show a view of GB2 in the Ni 12-nm HA sample. As in the previous case, the main crystallographic directions are indicated. The misfit between the [100] directions is about $50^{\circ}$. The common axis for this grain boundary was also close to a $\langle 110\rangle$ axis. This represents a deviation from a perfect $\Sigma=3$ twin of about $20^{\circ}$, which is outside the Brandon criterion range. This misorientation is not close to any other special boundary, and the boundary is therefore not considered to be a special boundary. As expected, no structural blocks of a $\Sigma=3$ twin are observed in this boundary. The grain boundary is, however, not amorphous. This is demonstrated in the figure where we show a view along a noncommon $\langle 110\rangle$ direction for the grain on the right-hand side. A significant degree of structural coherence across the grain-boundary plane can be observed, with the $\{111\}$ planes in the left grain continuing onto $\{311\}$ planes of the right grain. The misfit due to the different interplanar distance for these two types of planes is accommodated by a more disor- dered region every three planes. The maximum dimensions on the grain-boundary plane between these particular grains are approximately $12 \times 15 \mathrm{~nm}^{2}$ for the $12-\mathrm{nm}$ sample and 4 $\times 5 \mathrm{~nm}^{2}$ for the 5.2-nm sample. As mentioned above, using the Brandon criterion this boundary is outside the range of the $\Sigma=3$ special boundary and, indeed, we do not see the $\Sigma=3$ structural blocks, but we do see a significant degree of coherence in the structure. As in the previous boundaries analyzed, the basic features of the observed coherency and the accommodation of misfit are independent of grain size. Coherence in this context means a strong tendency for a family of planes of one grain to be continued by another family of plains in the neighboring grain. This is in general obtained through the alternation of highly ordered and highly disordered regions

Figure 4 shows another quite general boundary, GB4 in Ni 12-nm HA with a high-index common crystallographic axis. The grain on the left side of the grain-boundary plane is viewed along a $\langle 110\rangle$ axis. Significant structural coherency across the boundary can also be observed in this case, which corresponds to a misorientation of about $30^{\circ}$.

As our next example, in the Ni 8.0-nm HA sample, we found a grain boundary with misorientation of about $17^{\circ}$ around an axis close to $\langle 110\rangle$. The boundary plane is close to a $\{112\}$ type plane. Figure 5 shows a section perpendicular to the common $\langle 110\rangle$ direction. As in the previous cases, the unit cells of both grains are highlighted. There is again some degree of coherence between $\{111\}$ planes. To accommodate the misfit between the grains, a periodic array of $\frac{1}{2}\langle 110\rangle$ dislocations is formed, with dislocation lines also along $\langle 110\rangle$. In a front view of the grain-boundary plane, with the atoms that are fcc-coordinated removed, we see the dislocation lines and a small region of hcp-coordinated atoms in the dislocation core areas. We interpreted this region as a degree of dissociation of the $\frac{1}{2}\langle 110\rangle$ dislocation into Shockley partials. The dislocation lines are bound by the presence of other grain boundaries.

\section{B. Low-angle Cu sample}

To obtain a more general picture of grain boundaries in nanocrystalline materials and the possible influence of textures, we analyzed low-angle boundaries in the $\mathrm{Cu} 8.0-\mathrm{nm}$ LA sample.

The first example, shown in Figs. 6(a) and 6(b), is a boundary with a misfit of $12^{\circ}$. The boundary is clearly composed of a network of dislocations. Figure 6(a) shows a projection of the two grains, taken along a nearly common $\langle 110\rangle$ axis, and shows that for the grain on the right-hand side, the boundary plane is close to a $\{112\}$-type plane. In the grain on the left-hand side, the grain-boundary plane is rather close to a $\{111\}$-type plane. Figure 6(b) shows the two-dimensional network of dislocations that accommodate the misfit. There are a few hcp-coordinated atoms that can be observed in the core of the dislocations

A second example is the grain boundary shown in Fig. 7. The misorientation between the grains is $9^{\circ}$, as indicated in the figure, which shows a section along an axis close to a common $\langle 110\rangle$ axis. The misorientation is accommodated again by Shockley partial dislocations, generating stacking faults, which in this case form within the grain-boundary 


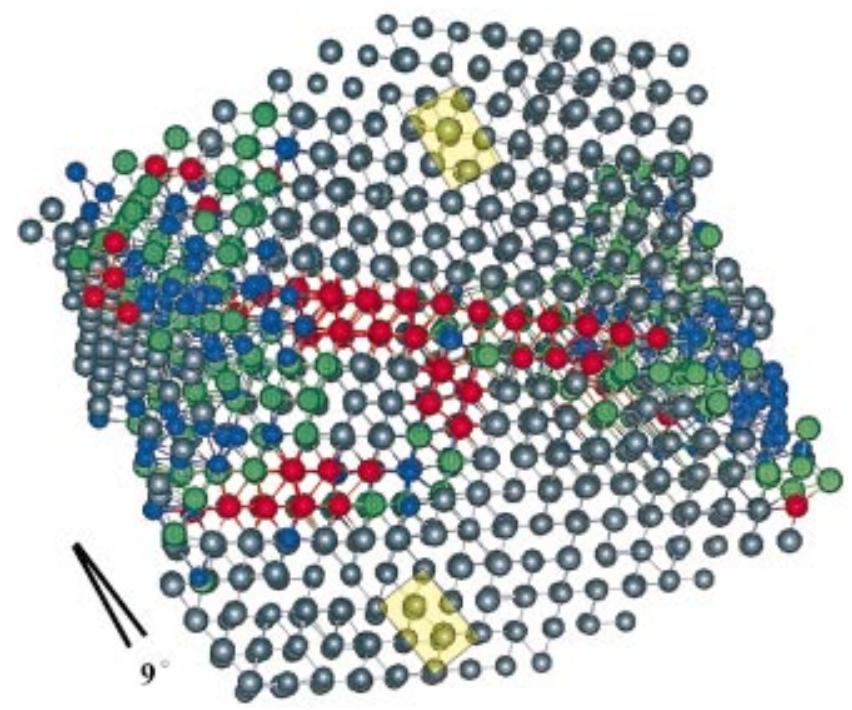

FIG. 5. (Color) Section of a grain boundary in Ni 8.0-nm HA with misorientation of about $17^{\circ}$ around an axis close to $\langle 110\rangle$. The boundary plane is close to a $\{112\}$ plane. The view is perpendicular to this $\langle 110\rangle$ direction. The unit cells are highlighted in each grain. To accommodate the misfit between the grains, a periodic array of $\frac{1}{2}\langle 110\rangle$ dislocations is formed, with dislocation lines along $\langle 110\rangle$. They present some splitting into Shockley partials. The color code is the same as in Fig. 2.

plane. The grain boundary is very small, about $2-3 \mathrm{~nm}$, and that is probably the reason why one stacking fault is sufficient to accommodate the grain-boundary misfit. However, the misfit is apparently not fully accommodated by the partial, because at a few atomic layers distance and parallel to the grain-boundary plane a stacking fault created by a second partial can be observed in one of the grains. Our observations for low-angle grain boundaries show a dislocation network

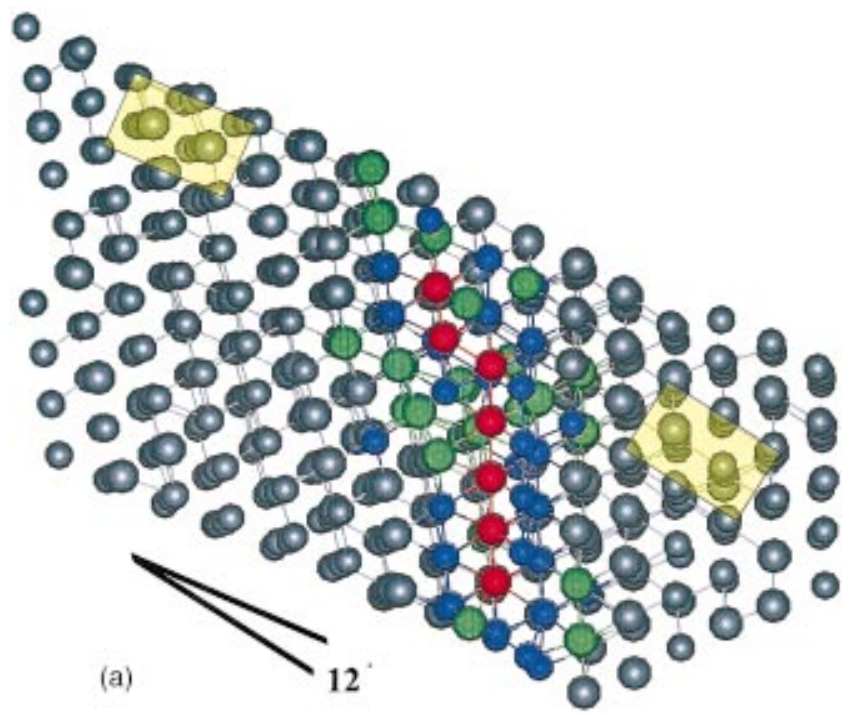

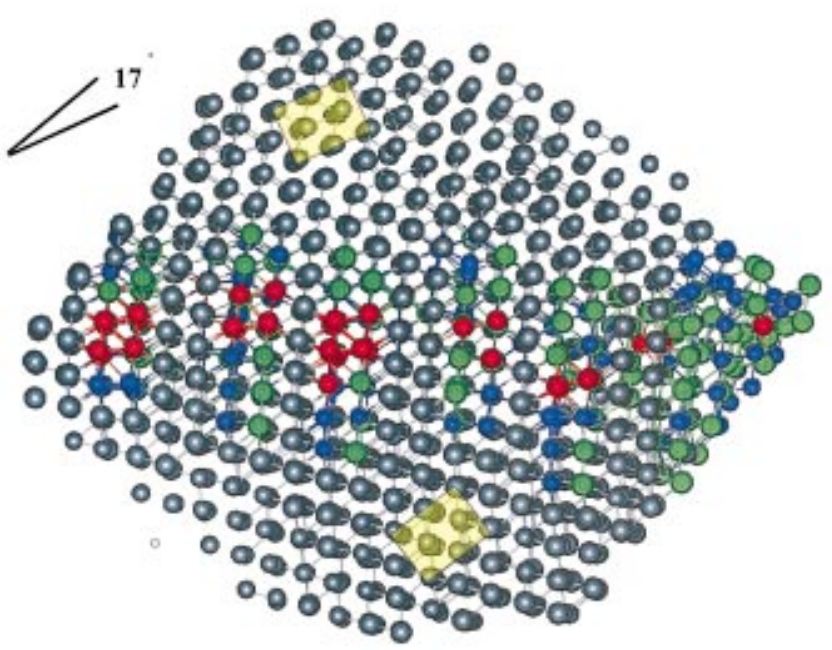

FIG. 7. (Color) View along an axis close to a common $\langle 110\rangle$ axis of the boundary as in Fig. 6. The misorientation is accommodated by Shockley partial dislocations, generating stacking faults, which in this case form within the grain-boundary plane. The unit cell is highlighted in each grain. The color code is the same as in Fig. 2 .

for the accommodation of the misfit, as expected for largergrain polycrystalline materials.

\section{Grain-boundary energies}

The simulations provide potential and kinetic energies for each atom and approximate grain-boundary energies can be calculated from these data. As a first level of approximation to the grain-boundary energy, we compare the energy of the entire sample to the energy of an equal number of perfect crystal atoms, both at $0 \mathrm{~K}$. This is the excess energy attributable to the grain boundaries present in the nanostructure.

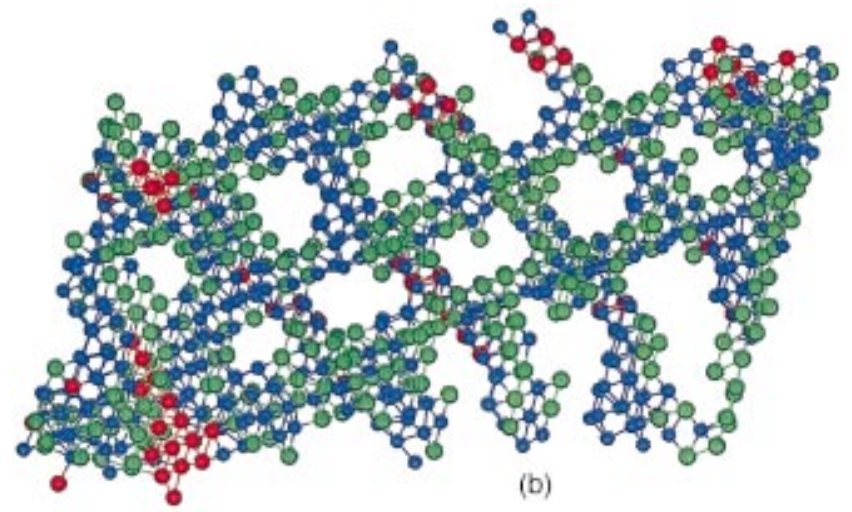

FIG. 6. (Color) A boundary with a $12^{\circ}$ misfit in the $\mathrm{Cu} 8.0-\mathrm{nm}$ LA sample. The boundary is composed of a network of dislocations. (a) A projection of the two grains along a nearly common $\langle 110\rangle$ axis; the boundary plane is close to a $\{112\}$-type plane. The grain-boundary plane is close to a $\{111\}$ orientation for the left grain. The unit cell is highlighted in each grain. (b) The two-dimensional network of dislocations that accommodate the misfit. There are a few hcp-coordinated atoms that can be observed in the dislocation core regions. The color code is the same as in Fig. 2. 
Dividing this excess energy by the average grain surface assuming spherical, equal-sized grains, we get $\gamma=1.8 \mathrm{~J} / \mathrm{m}^{2}$. Assuming cubic grains, we get a value for the average grain boundary energy of $1.4 \mathrm{~J} / \mathrm{m}^{2}$. More precise values that do not require an assumption for the geometric shape of the grains can be obtained for each particular boundary. In this technique, we define a volumetric region of the sample containing a given grain-boundary plane, far from its limits, that is, without including contributions from triple junction lines. The energy of the atoms in this region is compared to the perfect crystal energy of an equal number of atoms to obtain the grain-boundary energy. Using this procedure for GB1 (see Fig. 2), which contains structural units typical of a low-energy twin, we obtain a grain-boundary energy $\gamma$ $=1.1 \mathrm{~J} / \mathrm{m}^{2}$. For GB2 (Fig. 3), which is a much more disordered grain boundary, we obtain $\gamma=1.5 \mathrm{~J} / \mathrm{m}^{2}$. A similar value of $\gamma=1.6 \mathrm{~J} / \mathrm{m}^{2}$ is found for GB4 (Fig. 4). These numbers can be compared with experimental values obtained in differential scanning calorimetry (DSC) measurements. These are $1.1 \mathrm{~J} / \mathrm{m}^{2}$ in electroplated $\mathrm{Ni}$ with a $20-\mathrm{nm}$ grain size, ${ }^{39} 1.1 \mathrm{~J} / \mathrm{m}^{2}$ in a $8-\mathrm{nm}$ Pt sample synthesized by inert gas condensation, ${ }^{40}$ and $3 \mathrm{~J} / \mathrm{m}^{2}$ in a ball-milled Fe sample with mean grain size of $16 \mathrm{~nm} .^{41}$ The latter measurement was performed on an as-prepared (nonaged) sample, and the higher values are possibly due to the unrelaxed state of the grain boundaries. We note that these are mean values for the whole sample and are very sensitive to the definition of the geometrical factor used in the derivation of the grain boundary energies. ${ }^{41}$

\section{DISCUSSION AND CONCLUSIONS}

In the grain boundaries analyzed, we found that for misfits within a certain deviation from the perfect twin (within about $10^{\circ}$ ) the grain boundaries contain a repeating building structure consisting of portions of $\Sigma=3$ twin planes connected by more disordered regions or steps. In the $\mathrm{Ni} 12-\mathrm{nm}$ sample, these blocks are repeated several times within the grainboundary plane. The same structure was found in the 5.2-nm sample, with nearly no repetition due to size limitations. For larger deviation from the perfect twin or for other types of misfit, we always observed some kind of structural coherence. This coherence is manifested in a structure where a particular type of crystallographic planes in one grain is continued onto another type of plane in the other grain. The misfit caused by different interplanar spacing is accommodated by the presence of more disordered regions in a regular pattern. This is true even for general grain boundaries with high-index common axis.

For some of the boundaries studied, the atomic structure reveals clear evidence of dislocation networks, with some degree of decomposition of the dislocations into Shockley partials. Similar results are obtained for low-angle grain boundaries present in the textured sample. These dislocation networks are the usual expected mechanism of misfit accommodation in low-angle boundaries in large-grain polycrystals. These results were obtained in grain boundaries of total linear dimensions as small as $2 \times 3 \mathrm{~nm}^{2}$, providing evidence against the proposed model of these boundaries in terms of highly disordered, amorphous, or cementlike interfaces in nanophase bulk materials.

These conclusions are just opposite to those of a recent paper by Keblinski et al. ${ }^{22}$ on computer-simulated nanophase $\mathrm{Pd}$. In fact, by analyzing the pair distribution function (PDF) of those atoms with the highest potential energy, they conclude that the structure of the grain boundaries is a "glassy," gluelike phase, recalling the mentioned cementlike model. In several of our previous studies (see, for example, Fig. 3 in Ref. 42, Fig. 3 in Ref. 28, and Fig. 2 in Ref. 43), we have calculated the PDF's for atoms in the boundary selected through different criteria related either to the coordination or the energy. We find that the reminiscences of the secondneighbor peak, indicative of amorphous structure, are sensitive to the criteria used. The conclusion reached by Keblinski et al. is probably a consequence of the criterion they apply to select the atoms used to calculate the PDF, i.e., the atoms with the "highest potential energy." Those are the atoms that differ the most from the fcc coordination, as can be seen in Fig. 3 in Ref. 43 and Fig. 1 in Ref. 30. These figures show that approximately $20 \%$ of the atoms are non- 12 coordinated and have energy higher that the perfect crystal values plus the heat of melting, $180 \mathrm{meV}$ for this potential, used as the unit of energy needed to be in an amorphous environment. We believe that the PDF technique used to analyze the grainboundary structure can be misleading, with a high degree of coherence in the structure undetected. The atomistic structural visualization together with the bond analysis we report in this paper gives clear evidence supporting our conclusion. Our results agree with experimental evidence reported in literature that the grain boundaries in nanocrystalline materials are similar to those in conventional polycrystals.

Regarding the validity of these results with respect to the limitations of the empirical potential used, we have tested some samples with two other potentials, namely, from Baskes et l. $^{35}$ and from our own work, ${ }^{36}$ obtaining essentially the same structures. This is further confirmation that these structures are strongly dependent and largely determined by the geometry of the grain boundary. Also, the times used to equilibrate the structures at $300 \mathrm{~K}$ are long enough to arrive to metastable equilibrium. Longer relaxation, eventually at higher temperatures, can only lead to more ordered grain-boundary structures.

However, the presence of highly structured grain boundaries does not necessarily imply that grain-boundary dislocations control intergrain plasticity. Our previous observations that plasticity is controlled by an atomistic jump process, ${ }^{28}$ together with the present results, point towards the disordered regions in the grain boundaries as candidates for playing a primary role in the deformation process.

\section{ACKNOWLEDGMENTS}

This research was supported by the Swiss National Science Fund, Grant No. 21-46832.96, and partially by CONICET, Argentina, Grant No. PIP 0664/98. We acknowledge support from NSF Materials Theory and the NSFCONICET, U.S.-Argentina collaborative international program. 
${ }^{1}$ S. R. Agnew and J. R. Weertman, Mater. Sci. Eng., A 242, 174 (1998).

${ }^{2}$ R. Z. Valiev, I. V. Alexandrov, W. A. Chiou, R. S. Mishra, and A. K. Mukherjee, Mater. Sci. Forum 235-238, 497 (1997).

${ }^{3}$ S. R. Agnew, B. R. Elliot, C. J. Youngdahl, K. J. Hemker, and J. $\mathrm{R}$. Weertman, in Modelling of Structure and Mechanics from Microscale to Product, edited by J. V. Carstensen, T. Leffers, T. Lorentzen, O. B. Pedersen, B. F. Sorensen, and G. Winther (Risø National Laboratory, Roskilde, Denmark, 1998), p. 1.

${ }^{4}$ C. C. Koch, D. G. Morris, K. Lu, and A. Inoue, MRS Bull. 24, 54 (1999).

${ }^{5}$ H. Natter, M. Schmelzer, and R. Hempelmann, J. Mater. Res. 13, 1186 (1998).

${ }^{6}$ R. Weertman, D. Farkas, K. Hemker, H. Kung, M. Mayo, R. Mitra, and H. Van Swygenhoven, MRS Bull. 24, 44 (1999).

${ }^{7}$ A. P. Sutton and R. W. Balluffi, Interfaces in Crystalline Materials (Clarendon, New York, 1995).

${ }^{8}$ B. Chalmers, Physical Metallurgy (Wiley, New York, 1962).

${ }^{9}$ M. Bacia, J. Morillo, J. M. Penisson, and V. Pontikis, Philos. Mag. A 76, 945 (1997).

${ }^{10}$ D. L. Medlin, S. M. Foiles, and C. B. Carter, in Microscopy and Microanalysis, edited by G. W. Bailey, J. M. Corbett, R. V. W. Dimlich, J. R. Michael, and N. J. Zaluzec (San Francisco Press, San Francisco, 1996).

${ }^{11}$ I. Majid, C. A. Counterman, P. D. Bristowe, and R. W. Balluffi, Acta Metall. Mater. 42, 3331 (1994).

${ }^{12}$ D. N. Seidman, J. G. Hu, S. M. Kuo, D. W. Krakauer, Y. Oh, and A. Seki, J. Phys. (Paris), Colloq. XX, C1-47 (1990).

${ }^{13}$ K. L. Merkle and D. Wolf, in Structural Property Relationships for Metal/Metal Interfaces, edited by A. D. Romig, D. E. Fowler, and P. D. Bristowe, MRS Symposia Proceedings No. 229 (Materials Research Society, Pittsburgh, 1991), p. 185.

${ }^{14}$ J. Erhart and V. Paidar, Mater. Sci. Forum 294, 381 (1999).

${ }^{15}$ P. G. Sanders, J. A. Eastman, and J. R. Weertman, Acta Mater. 46, 4195 (1998).

${ }^{16}$ Y. Champion and M. J. Hytch, Appl. Phys. (N.Y.) 4, 161 (1998).

${ }^{17}$ E. A. Stern, R. W. Siegel, M. Newville, P. G. Sanders, and D. Haskel, Phys. Rev. Lett. 75, 3874 (1995).

${ }^{18}$ S. C. Mehta, D. A. Smith, and U. Erb. Mater, in Molecularly Designed Ultrafine/Nanostructures Materials, edited by K. E. Gonsalves et al., MRS Symposia Proceedings No. 351 (Materials Research Society, Pittsburgh, 1994), p. 337.

${ }^{19}$ J. Loffler and J. Weissmuller, Phys. Rev. B 52, 7076 (1995).

${ }^{20}$ Y. H. Zhao, K. Lu, and T. Liu, Phys. Rev. B 59, 11117 (1999).
${ }^{21}$ K. Lu and N. X. Sun, Philos. Mag. Lett. 75, 389 (1997).

${ }^{22}$ P. Keblinski, D. Wolf, S. R. Phillpot, and H. Gleiter, Scr. Mater. 41, 631 (1999).

${ }^{23}$ H. Zhu and R. S. Averback, Philos. Mag. Lett. 73, 27 (1996).

${ }^{24}$ C. L. Liu, J. B. Adams, and R. S. Siegel, Nanostruct. Mater. 4, 265 (1994).

${ }^{25}$ S. R. Phillpot and D. Wolf, J. Appl. Phys. 78, 847 (1995).

${ }^{26}$ See K. T. Aust and B. Chalmers, in Metal Interfaces (ASM, Cleveland, 1952), p. 153.

${ }^{27}$ H. Van Swygenhoven and A. Caro, Appl. Phys. Lett. 71, 1652 (1997).

${ }^{28}$ H. Van Swygenhoven and A. Caro, Phys. Rev. B 58, 11246 (1998).

${ }^{29}$ H. Van Swygenhoven, M. Spaczer, D. Farkas, and A. Caro, Phys. Rev. B 60, 22 (1999).

${ }^{30}$ H. Van Swygenhoven, M. Spaczer, and A. Caro, Acta Mater. 47, 3117 (1999).

${ }^{31}$ G. Z. Voronoi, J. Reine Angew. Math. 134, 199 (1908).

${ }^{32}$ H. Van Swygenhoven, M. Spaczer, and A. Caro, Nanostruct. Mater. 12, 629 (1999).

${ }^{33}$ F. Cleri and V. Rosato, Phys. Rev. B 48, 22 (1993).

${ }^{34}$ M. Parrinello and A. Rahman, J. Appl. Phys. 52, 7182 (1981).

${ }^{35}$ M. I. Baskes, X. Sha, J. E. Angelo, and N. R. Moody, Modell. Simul. Mater. Sci. Eng. 5, 651 (1997).

${ }^{36}$ Y. Mishin, D. Farkas, M. J. Mehl, and D. A. Papaconstantopoulos, Phys. Rev. B 59, 5 (1999); D. J. Honneycutt and H. C. Andersen, J. Phys. Chem. 91, 4950 (1987).

${ }^{37}$ D. J. Honneycutt and H. C. Andersen, J. Phys. Chem. 91, 4950 (1987).

${ }^{38}$ D. G. Brandon, Acta Metall. 14, 1479 (1966).

${ }^{39}$ U. Klement, U. Erb, and K. T. Aust, Nanostruct. Mater. 6, 581 (1995); A. Tschope, R. Birringer, and H. Gleiter, J. Appl. Phys. 71, 5391 (1992).

${ }^{40}$ A. Tschöpe, R. Birringer, and H. Gleiter, J. Appl. Phys. 71, 5391 (1992).

${ }^{41}$ C. H. Moelle and H. J. Fecht, Nanostruct. Mater. 6, 421 (1995).

${ }^{42}$ H. Van Swygenhoven, M. Spaczer, and A. Caro, in Microscopic Simulation of Interfacial Phenomena in Solids and Liquids, edited by S. R. Phillpot, P. D. Bristowe, D. G. Stroud, and J. R. Smith, MRS Symposia Proceedings No. 492 (Materials Research Society, Pittsburgh, 1998), p. 357.

${ }^{43}$ H. Van Swygenhoven, M. Spaczer, and A. Caro, Nanostruct. Mater. 10, 819 (1998). 